\title{
Biodegradación del explosivo tetranitrato de pentaeritritol (PETN) por bacterias aisladas de ambientes mineros
}

\author{
Biodegradation of the explosive pentaerythritol tetranitrate (PETN) by bacteria isolated from \\ mining environments
}

Yerson Durán, Christian Andrade, Armando Martínez, Tito Sánchez, Fernando De la Cruz, Pablo Ramírez*

Laboratorio de Microbiología Molecular y Biotecnología, Facultad de Ciencias Biológicas, Universidad Nacional Mayor de San Marcos, Perú. Apartado postal 110058, Lima 11, Perú.

*Autor para correspondencia Pablo Ramírez E-mail: pramirezr@unmsm.edu.pe

Email Yerson Durán: yersonduran26@gmail.com

Email Christian Andrade: chris_bio571@hotmail.com

Email Armando Martínez: biologia.b10@hotmail.com

Email Tito Libio Sànchez: bioambiente@hotmail.com

EmailFemandoDelaCruz: fernandodelacruzcalvo@gmail.com

Citación:

Durán Y., C. Andrade, A. Martínez, T. Sánchez, F. De la Cruz \& P. Ramírez. 2013. Biodegradación del explosivo tetranitrato de pentaeritritol (PETN) por bacterias aisladas de ambientes mineros. Rev. peru. biol. 20(2): 145 - 150 (Diciembre 2013)

\section{Resumen}

El tetranitrato de pentaeritritol (PETN) es el detonante más energético y recalcitrante empleado en la actividad minera ya que posee cuatro grupos nitro unidos mediante enlaces éster; además es un contaminante orgánico persistente según el Convenio de Estocolmo. En el presente trabajo es evaluada la eficiencia de degradación del PETN por bacterias aisladas de ambientes mineros. En primer lugar se seleccionó las cepas con mayor crecimiento durante tres subcultivos en medio mínimo con PETN como única fuente de nitrógeno. Luego, se identificó mediante análisis del gen RNAr $16 S$ las cepas Bacillus sp. J8A2 y Enterobacter $\mathrm{sp}$. M1B. Las velocidades específicas de crecimiento fueron de $0.057 \mathrm{~h}^{-1}$ para J8A2 y 0.042 $\mathrm{h}^{-1}$ para M1B, y las velocidades específicas de degradación de 0.72 y $1.16 \mathrm{mmol}$ de PETN/g de proteína.h, respectivamente. Además, el extracto crudo de la cepa J8A2 mostró una actividad específica $(U)$ de $1.03 \mathrm{mmol}$ de PETN/h y una actividad específica por proteína $(\mathrm{U} / \mathrm{g})$ de $138.6 \mathrm{mmol}$ de PETN/g.h.

Palabras clave: Tetranitrato de pentaeritritol; contaminación; minería; biodegradación; Bacillus; Enterobacter.

\section{Abstract}

Pentaerythritol tetranitrate (PETN) is the most energetic and recalcitrant detonating used in mining activity being that it has four nitro groups linked by ester bounds, also is considered a persistent organic pollutant in agreement with the Stockholm Convention. In this work the degradation efficiency of PETN by bacteria isolated from mining environments is evaluated. At first, strains that showed growth during three subcultures with PETN as unique source of nitrogen were selected. Before, we identified Bacillus sp. J8A2 and Enterobactersp. M1B by the 16S rRNA gene partial sequences analysis. Specific growth rate were $0.057 \mathrm{~h}-1$ for J8A2 and $0.042 \mathrm{~h}-1$ for M1B, and specific rate of PETN degradation were 0.72 and $1.16 \mathrm{mmol}$ of PETN/g of protein.h respectively. Moreover, crude extract of J8A2 strain showed a specific activity (U) of $1.03 \mathrm{mmol}$ of PETN/h and a specific activity per protein $(\mathrm{U} / \mathrm{g})$ of $138.6 \mathrm{mmol}$ of PETN/g.h.

Keywords: pentaerythritol tetranitrate; contamination; mining; biodegradation; Bacillus; Enterobacter.

\section{Introducción}

La contaminación por explosivos es una preocupación muy importante en los lugares donde son producidos y almacenan, pero sobretodo donde se emplean (Binks et al. 1996, French et al. 1998, Ibeanusi et al. 2009, Singh et al. 2012), porque no son comunes en el ambiente y son recalcitrantes. Entre los explosivos más energéticos está el tetranitrato depentaeritritol (PETN), el cual se sintetiza mediante la nitrificación de derivados de alcoholes formando enlaces éster de difícil degradación (White et al. 1996). Además, su estabilidad en el ambiente a $30^{\circ} \mathrm{C}$ expresado en vida media extrapola un valor de 12 millones de años (Fotlz 2012).

El PETN está clasificado dentro del Grupo A de explosivos primarios o iniciadores según la clasificación del sistema UN (Naciones Unidas) y ha sido designado con el código UN 150 y clasificado de riesgo 1.1D (Global security 2011). Es un detonante que no reacciona por movimiento brusco o la ignición a presión atmosférica normal, sino que es sensible a la fricción, más que otros explosivos como el 2,4,6 trinitrotolueno

(TNT) y el tetril (Jaw \& Lee 2008). Sin embargo, la reactividad del PETN no está $\begin{array}{ll}\text { Presentado: } & 11 / 04 / 2013 \\ \text { Aceptado: } & 28 / 09 / 2013\end{array}$

$\begin{array}{ll}\text { Aceptado: } & \text { 28/09/2013 } \\ \text { Publicado online: } & 09 / 12 / 2013\end{array}$

( ) Los autores. Publicado por la Revista Peruana de Biología de la Facultad de Ciencias Biológicas, Universidad Nacional Mayor de San Marcos. Este es un artículo de acceso abierto, distribuido bajo los términos de la Licencia de Atribución Reconocimiento-NoComercial-SinObraDerivada 3.0 de Creative Commons (http://creativecommons.org/licenses/by-nc-nd/3.0/deed. es_ES), que permite el uso no comercial, distribución y reproducción en cualquier medio, siempre que la obra original sea debidamente citadas. Para uso comercial, por favor póngase en contacto con editor.revperubiol@gmail.com. 
asegurada por sus características químicas, por lo que algunos dispositivos explosivos en la industria minera o petrolera pueden no explosionar por problemas técnicos y constituirse en un riesgo para el ambiente y las personas (Nyanhongo et al. 2008).

Las características recalcitrantes, xenobióticas y el riesgo para la salud del hombre y el ambiente que causa la contaminación por PETN fue contemplado en el Convenio de Estocolmo sobre Contaminantes Orgánicos Persistentes (COP's), y donde el Perú es parte comprometida a tomar medidas jurídicas y administrativas para la eliminación de estos contaminantes (SINIA 2007).

En respuesta a este problema global se han estudiado microorganismos degradadores de PETN como Enterobacter cloacae PB2 y Agrobacterium radiobacter, los cuales son capaces de degradar otros explosivos como el TNT y sus derivados (French et al. 1998), y que han sido reportados como carcinogénicos (Esteve-Núñez et al. 2001). Es así que microorganismos capaces de degradar ambos compuestos serían los más efectivos durante el empleo del pentolito (mezcla de TNT y PETN), cuyo uso es común en nuestro país cuando se emplea ANFO (mezcla de nitrato de amonio y combustible) para voladuras (Estudios mineros, 2011).

En este contexto, en el presente trabajo se informa de la selección de bacterias degradadoras de PETN aisladas de ambientes mineros, su identificación molecular y la determinación de su eficiencia en la degradación de PETN, con la perspectiva de desarrollar técnicas de control de contaminación y riesgos por explosivos en el Perú.

\section{Material y métodos}

Material biológico.- Diecinueve cepas bacterianas del cepario del Laboratorio de Microbiología Molecular y Biotecnología de la Facultad de Ciencias Biológicas de la Universidad Nacional Mayor de San Marcos fueron evaluadas. Las cepas fueron aisladas de ambientes mineros de las regiones La Libertad, Junín y Lima.

Selección de cepas que degradan PETN.- Las cepas fueron cultivadas durante $12-16$ horas en caldo TSB a $28^{\circ} \mathrm{C}$ y lavadas en solución salina al $0.85 \%$. Se inoculó al $0.5 \%$ en Caldo PETN (Binks et al. 1996, White et al. 1996) compuesto por (g/L): $\mathrm{K}_{2} \mathrm{HPO}_{4} 3.5, \mathrm{KH}_{2} \mathrm{PO}_{4} 1.5, \mathrm{MgSO}_{4} 0.124, \mathrm{NaCl} 0.5$, glucosa 10 y PETN 0.316 como única fuente de nitrógeno y $0.73 \mathrm{~mL} / \mathrm{L}$ de glicerol y el pH fue ajustado a 7.02; se adicionó $1 \mathrm{~mL} / \mathrm{L}$ de solución de trazas al medio de cultivo (White et al. 1996). Se incubó a $28{ }^{\circ} \mathrm{C}$ a $200 \mathrm{rpm}$ por 7 días. Al cabo de los 7 días se midió el crecimiento por densidad óptica a $600 \mathrm{~nm}\left(\mathrm{DO}_{600}\right)$ y se verificó su pureza en agar tripticasa soya (TSA) y agar mínimo $\mathrm{P}$ compuesto por $(\mathrm{g} / \mathrm{L}): \mathrm{K}_{2} \mathrm{HPO}_{4} 3.5, \mathrm{KH}_{2} \mathrm{PO}_{4} 1.5, \mathrm{MgSO}_{4}$ $0.124, \mathrm{NaCl} 0.5$, glucosa $10, \mathrm{NH}_{4} \mathrm{NO}_{3} 0.24$, pH 7.02 y agaragar al $1.5 \%$. Se seleccionaron las cepas que mostraron mayor densidad óptica. Para determinar las características fenotípicas y moleculares se siguió lo establecido en el Manual de Bacteriología Sistemática de Bergey (Brenner et al. 2005, De Vos et al. 2009).

Amplificación del gen $R N A r$ 16S.- Para la extracción de DNA genómico, las cepas fueron cultivadas en caldo TSB por 12-16 horas a $28{ }^{\circ} \mathrm{C}$ y se aplicó el protocolo de purificación del Wizard Genomic DNA Purification Kit (Promega). Para la PCRse emplearon los iniciadores 27F (5'-AGAGTTTGATCMTGGCTCAG-3') y 519R (5'-GWATTACCGCGGCKGCTG-3') para amplificar las $500 \mathrm{pb}$ correspondiente al extremo 5' del gen $R N A r 16 S$ (Weisburg et al. 1991). En la
PCR se emplearon las indicaciones de la KOD HOT start DNA polymerase (Novagen ${ }^{\circ}$ y el programa fue: desnaturalización inicial $95^{\circ} \mathrm{C}$ por 2 minutos, 33 ciclos de desnaturalización a $95^{\circ} \mathrm{C}$ por 30 segundos, hibridación a $62{ }^{\circ} \mathrm{C}$ por 10 segundos, amplificación a $70{ }^{\circ} \mathrm{C}$ por 10 segundos, y amplificación final a $70{ }^{\circ} \mathrm{C}$ por 10 minutos. Los productos de PCR fueron evidenciados en geles de agarosa $1 \%$ en tampón TAE $0.5 \mathrm{X}$, teñidas con bromuro de etidio al $0.5 \%$ y reveladas en transiluminador UV. Se empleó como marcador de peso molecular $1 \mathrm{~kb}$ plus DNA Ladder (Promega ). El secuenciamiento parcial del gen $R N A r 16 S$ se realizó según el protocolo del BigDye $e^{\circledast}$ Terminator v3.1 Cycle Sequencing Kit en el secuenciador Automatic Sequencer 3730xl por MACROGEN KOREA.

Determinación de la relación filogenética.- Las secuencias consenso del gen $R N A r 16 S$ de las cepas seleccionadas fueron comparadas con la Base de Datos del GenBank/EMBL/DDBJ usando la plataforma del programa local de alineamiento de secuencias BlastN versión 2.0 (Altschul et al. 1990). Luego se realizó un alineamiento múltiple con el programa Bioedit v7.0.9 (Hall 1999) con secuencias que presentaron similitud mayor o igual a 97\%, además de secuencias de otras especies relacionadas. Finalmente, se infirió el árbol filogenético con el programa Mega v5.2 (Kumar et al. 2008) empleando el modelo Neighbor-Joining, el método de substitución nucleotídica Maximum Composite Likelihood y 1000 réplicas de Bootstraps (Tamura et al. 2011).

Cinética de degradación de PETN.- Los ensayos se realizaron por triplicado en Caldo PETN modificado compuesto (g/L): $\mathrm{K}_{2} \mathrm{HPO}_{4} 1.68, \mathrm{MgSO}_{4} 0.124, \mathrm{NaCl} 0.5$, succinato $6.80, \mathrm{KOH}$ 7.5 , PETN 0.316 y Tween 80 al $0.1 \%$, y se incubó durante 82 horas a $28^{\circ} \mathrm{C}$ y $200 \mathrm{rpm}$. Se tomó muestras cada 12 horas para cuantificar PETN residual y proteínas totales como se describe a continuación.

Cuantificación de PETN residual y proteínas totales.- Se cuantificó el PETN residual según el Método Oficial 964.25 de la AOAC (2002): tetranitrato de pentaeritritol en medicamentos. Brevemente, a $1 \mathrm{~mL}$ de cultivo se adicionó $99 \mathrm{~mL}$ de ácido acético glacial y $4 \mathrm{~mL}$ de ácido fenoldisulfónico, se homogenizó y dejó en reposo por 30 minutos en oscuridad. Luego, se adicionó $60 \mathrm{~mL}$ de agua destilada y $15 \mathrm{~mL}$ de hidróxido de amonio concentrado, se agitó suavemente y se dejó enfriar, se midió la absorbancia a $408 \mathrm{~nm}$.

Para la cuantificación de proteínas totales, se mezcló $1 \mathrm{~mL}$ de cultivo con $1 \mathrm{~mL}$ de acetona y se centrifugó a $13000 \mathrm{rpm}$ por 3 minuto, luego se repitió este procedimiento en $100 \mu \mathrm{L}$ de acetona y se resuspendieron las células en hidróxido de sodio 0.1 M y se incubó a $80^{\circ} \mathrm{C}$ por 5 minutos (Trott et al. 2003). Seguido, se empleó el método de Bradford (1976) para la cuantificación de proteínas totales.

Cuantificación de la actividad PETN reductasa.- Se obtuvieron extractos crudos de las cepas en fase exponencial tardía cultivadas en caldo PETN modificado. Luego, se colectaron las células a $4500 \mathrm{rpm}$ por 10 minutos y se empleó tampón bis-tris propano $(50 \mathrm{mM}, \mathrm{pH} 7.0)$ para lavar y resuspender las células. Luego éstas fueron lisadas por sonicación por tres veces a 15 segundos alternados con 15 segundos de enfriamiento en hielo. Finalmente, se centrifugó a $13000 \mathrm{rpm}$ por 10 minutos a temperatura ambiente y se filtró el sobrenadante en membrana de nitrocelulosa de $0.45 \mathrm{~nm}$ (Binks et al. 1996). 


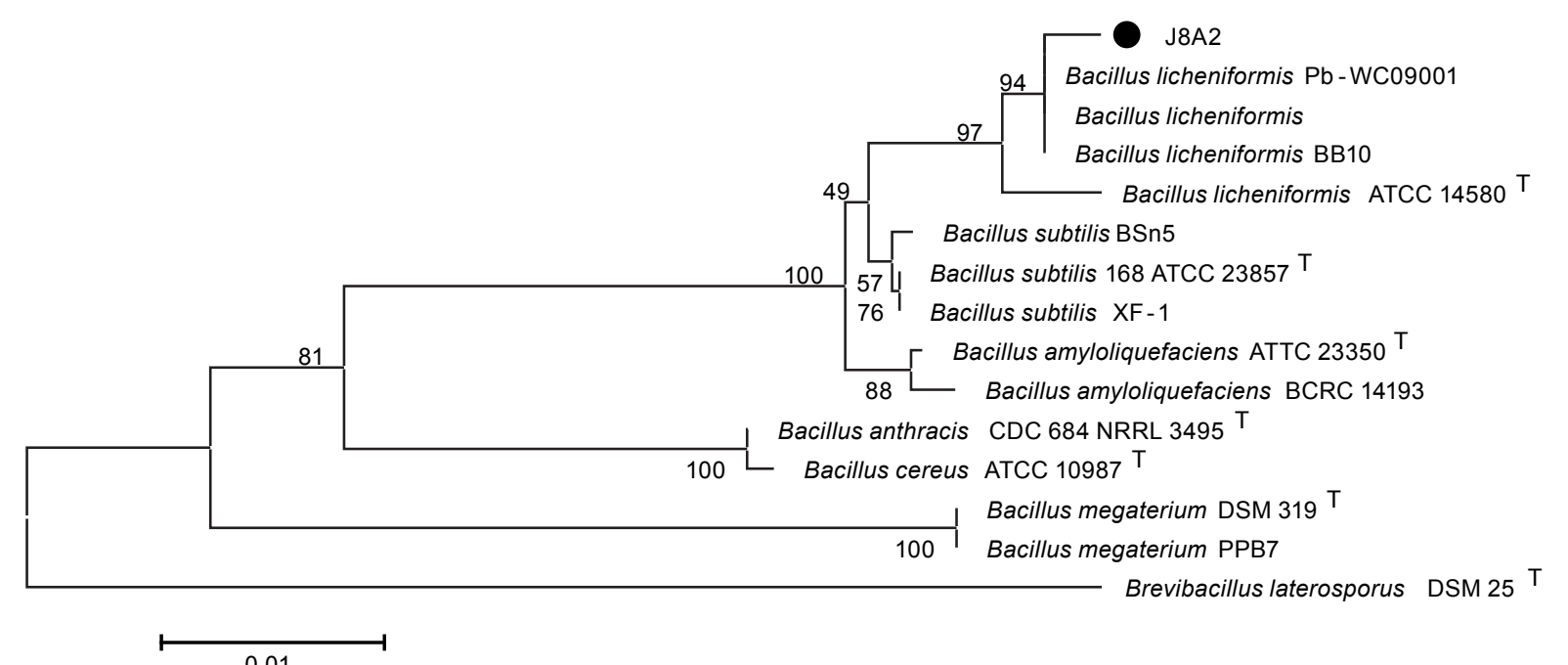

0.01

Figura 1. Relación filogenética de la cepa J8A2 (HF568785.1) (•) inferida a partir de 491pb del gen RNAr 16S aplicando el modelo NeighborJoining, el método de substitución nucleotídica Maximum Composite Likelihood con 1000 réplicas de Bootstraps. La cepa J8A2 conforma un clado con la especie Bacillus licheniformis. La barra representa el porcentaje de substituciones nucleotídicas. El símbolo ${ }^{\top}$ señala especie tipo.

Posteriormente, para medir la actividad PETN reductasa se empleó un volumen final de reacción de 3:2 mL del extracto crudo filtrado, $1 \mathrm{~mL}$ de tampón bis-tris propano ( $\mathrm{pH}$ 7.0), 0.2 mM de NADPH (concentración final) y $760 \mu \mathrm{M}$ de PETN (6 $\mu \mathrm{L}$ de $380 \mathrm{mM}$ PETN en acetona). La reacción se llevó a cabo en baño maría a $28^{\circ} \mathrm{C}$ por 45 minutos. A continuación se realizó la inactivación del NADPH por adición de cianuro férrico 0.5 $\mathrm{mM}$ y metosulfato de fenazina $0.2 \mathrm{mM}$ concentración final, seguidamente se midió la producción de nitritos mediante la reacción de Griess (Binks et al. 1996). La oxidación de NADPH fue monitoreada a $350 \mathrm{~nm}$ al inició y al final de la reacción y la cantidad de proteína en el filtrado se cuantificó mediante la reacción de Bradford (1976). Para realizar los cálculos una unidad de actividad enzimática fue definida como la cantidad de $\mu$ moles de NADPH oxidado por minuto y se consideró que por cada molécula de PETN se liberaban dos moléculas de nitrito basado en los resultados obtenidos por Binks et al. (1996).

\section{Resultados}

Cepas degradadoras de PETN.- De las 19 cepas evaluadas se seleccionaron la J8A2 y M1B como las más eficientes en la degradación de PETN. La cepa J8A2 mostró $\mathrm{DO}_{600}$ de 0.173 , 0.146 y 0.141 durante tres subcultivos, mientras que la M1B mostró $\mathrm{DO}_{600}$ de $0.132,0.120$ y 0.125 , respectivamente.

Análisis filogenético.- La secuencia consenso del gen $R N A r$ $16 S$ de J8A2 (HF568785.1) fue comparada con la Base de Datos del GenBank/EMBL/DDBJ mediante el programa BLASTn 2.0, y se obtuvo como resultado una identidad de $99 \%$ y una similitud de 97\% con Bacillus licheniformis Pb-WC09001 (HM006901.1), B. licheniformis BB10 (JN391533.1), B. licheniformis (AY479984.1) y con otras secuencias del género Bacillus no cultivables. Estas secuencias del gen RNAr $16 S$ de Bacillus licheniformis ATCC 14580 (CP000002.3), Bacillus subtilis 168 ATCC 23857 (AL009126.3), Bacillus subtilis XF-1 (P004019.1), Bacillus subtilis BSn5 (CP002468.1), Bacillus amyloliquefaciens ATTC 23350 (FN597644.1), Bacillus amyloliquefaciens BCRC 14193 (EF433408.1), Bacillus anthracis CDC684 NRRL 3495 (CP001215.1), Bacillus cereus ATCC 10987 (AE017194.1), Bacillus megaterium DSM319
(CP001982.1), Bacillus megaterium PPB7 (HM771662.1) y Brevibacillus laterosporus DSM 25 (AB112720.1) como grupo externo fueron alineadas y analizadas con el programa Mega 5.2 para inferir la respectiva relación filogenética en el cual se denota que la cepa J8A2 conforma un clado con $94 \%$ de probabilidad con la especie Bacillus licheniformis (Fig. 1).

Por otro lado, el análisis de la secuencia parcial del gen $r R N A$ $16 S$ de la cepa M1B (HF568786.1) con el programa BLASTn 2.0 mostró $100 \%$ de identidad y $95 \%$ de similitud con Enterobacter asburiae KNUC5007 (JQ682630.1), Enterobacter sp. Gg10 (JN020643.1), Enterobacter sp. AAP4 (JF276427.1), así como con secuencias de Enterobacter no cultivables. Dado que el análisis del gen $R N A r 16 S$, generalmente no discrimina especies dentro de la familia Enterobactereaceae (De Angelis et al. 2011) no fue imprescindible realizar el análisis filogenético de la cepa M1B.

Características fenotípicas.- La cepa J8A2 presenta las siguientes características fenotípicas a $28^{\circ} \mathrm{C}$ : bacilo corto Gram negativo (24 horas en agar TSA), motil, utiliza citrato como única fuente carbono, no hidroliza almidón, producción de indol negativo, sin producción de $\mathrm{H}_{2} \mathrm{~S}$, fermentación de glucosa con producción de acidez y gas, crecimiento en anaerobiosis, crecimiento en $\mathrm{NaCl}$ al 7\%, rojo de metilo positivo y VogesProskauer negativo. Características culturales de colonia en agar TSA a $28^{\circ} \mathrm{C}$ por 24 horas: plana, translúcida, con centro amarillo claro, sin pigmento difusible, borde irregular y un promedio de $3.0 \mathrm{~mm}$ de diámetro. Además, en algunas ocasiones presentó la apariencia de un cultivo mixto con colonias de borde regular, convexas, mucoides y opacas.

Por otro lado, la cepa M1B presentó las siguientes características fenotípicas a $28{ }^{\circ} \mathrm{C}$ : bacilo Gram negativo $(24$ horas en agar TSA), móvil, citrato negativo, indol negativo, lisina descarboxilasa, arginina y ornitina dehidrolasa, sin producción de $\mathrm{H}_{2} \mathrm{~S}$, fermentación de glucosa con producción de acidez y sin gas, rojo de metilo positivo débil y Voges-Proskauer negativo. Características culturales de colonia en agar TSA a $28{ }^{\circ} \mathrm{C}$ por 24 horas: convexa, blanca opaca, sin pigmento difusible, borde regular, lisas y un promedio de $2 \mathrm{~mm}$ de diámetro. 


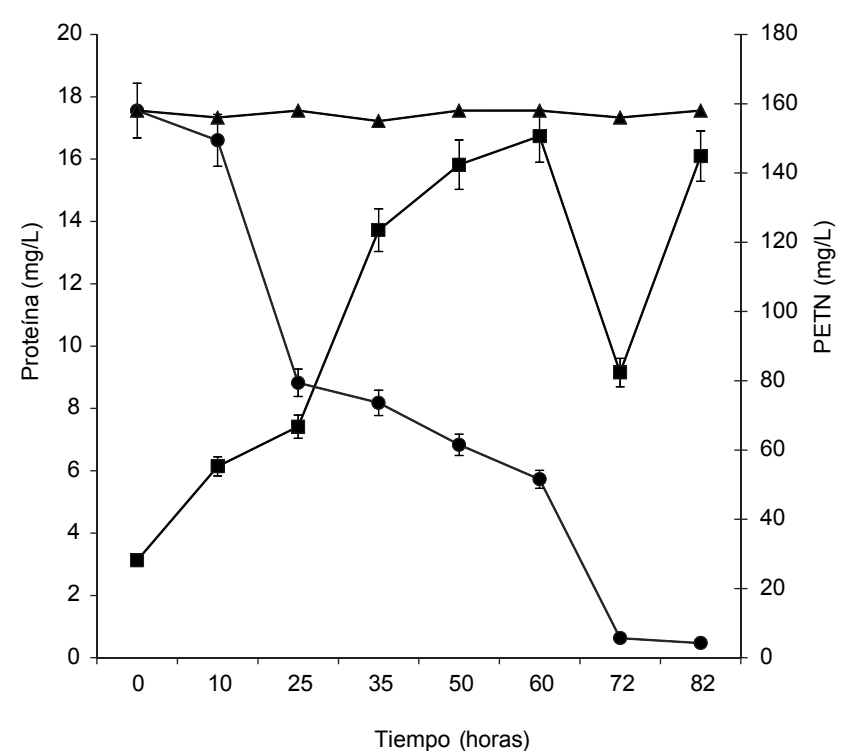

Figura 2. Cinética de degradación de PETN por Bacillus sp. J8A2 incubado por triplicado a $28^{\circ} \mathrm{C}$ y $200 \mathrm{rpm}$ en caldo PETN modificado. Concentración de PETN residual $(\bullet)$, concentración de proteína total $(\boldsymbol{\bullet})$, concentración de PETN en caldo sin inocular $(\boldsymbol{\Delta})$. Barras verticales: error estándar del promedio de tres repeticiones.

Cinética de degradación del PETN.- Con este fin se optimizó la composición del caldo PETN modificado recomendado por Park et al. (2003) (resultados no publicados). Las cepas J8A2 (Fig. 2) y M1B (Fig. 3) mostraron velocidades específicas de crecimiento de $0.057 \mathrm{~h}^{-1}$ y $0.042 \mathrm{~h}^{-1}$ respectivamente. La concentración de PETN se redujo al $50 \%$ en menos de 35 horas, obteniéndose un rendimiento específico de degradación de 1.16 mmol de PETN/g de proteína.h para M1B y $0.72 \mathrm{mmol}$ de PETN/g de proteína.h para J8A2; y un rendimiento por sustrato $21.56 \pm 1.5 \mathrm{~g}$ de proteína/mol de PETN para M1B y de 39.7 $\pm 2.1 \mathrm{~g}$ de proteína/mol de PETN para J8A2.

Después de 60 horas de cultivo se registró la mayor concentración de proteína de $16.74 \mathrm{~g} / \mathrm{L}$ para ambas cepas, sin embargo la cepa M1B presentó valores de $4.27 \mathrm{~g} / \mathrm{L}$ de PETN residual en comparación con J8A2 que mostró $51.56 \mathrm{~g} / \mathrm{L}$ de PETN residual. Por otro lado, a las 72 horas de la cinética se observó la reducción en la cantidad de proteína, a $7.96 \mathrm{~g} / \mathrm{L}$ para J8A2 y $9.15 \mathrm{~g} / \mathrm{L}$ para M1B. Posteriormente, a las 82 horas se registró un nuevo incremento de la concentración de proteína y la menor concentración de PETN, $2.12 \mathrm{mg} / \mathrm{L}$ para M1B y $4.25 \mathrm{mg} / \mathrm{L}$ para J8A2. Adicionalmente, a los 5 días de incubación se observó que el medio de cultivo de la cepa J8A2 viró a amarillo.

Actividad PETN reductasa del extracto crudo.- La velocidad específica de liberación de nitritos obtenida fue 4.6 $\mu \mathrm{mol} / \mathrm{min}$.mg a partir de $1.54 \mu \mathrm{mol}$ de nitritos liberados por $7.43 \mu \mathrm{g}$ de proteína total en 45 minutos y a $28^{\circ} \mathrm{C}$; y se registró la oxidación de $1.59 \mu \mathrm{mol}$ de NADPH calculándose una velocidad específica de oxidación de NADPH de 4.7 umol/ min.mg. Por otro lado, los controles sin NADPH registraron valores inferiores de liberación de nitritos con un promedio de $1.5 \mu \mathrm{mol} / \mathrm{min} . \mathrm{mg}$.

Considerando que se libera dos moléculas de $\mathrm{NO}^{-2}$ de cada molécula de PETN se calculó que $1.54 \mu \mathrm{mol}$ de nitritos fueron liberados a partir de $0.77 \mu \mathrm{mol}$ de PETN. Por lo tanto, la acti-

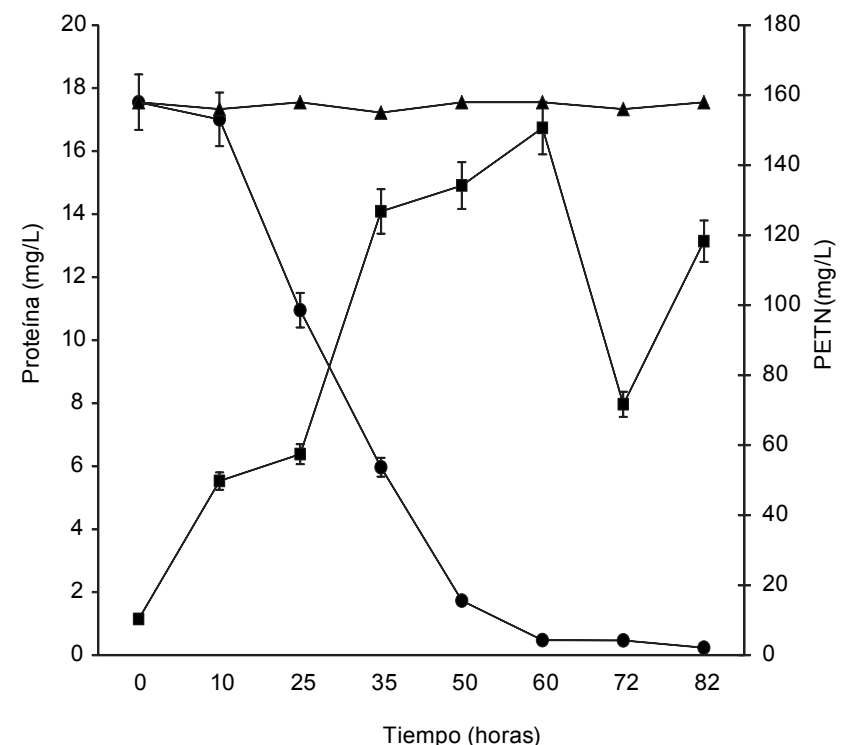

Figura 3. Cinética de degradación de PETN por Enterobactersp. M1B incubado por triplicado a $28^{\circ} \mathrm{C}$ y $200 \mathrm{rpm}$ en caldo PETN modificado. Concentración de PETN residual $(\bullet)$, concentración de proteína total $(\bullet)$, concentración de PETN en caldo sin inocular $(\boldsymbol{\Delta})$. Barras verticales: error estándar del promedio de tres repeticiones.

vidad específica (U) fue de $1.03 \mathrm{mmol}$ de PETN/h a partir de $7.43 \mathrm{mg}$ de proteína total y la actividad específica por proteína (U/g) fue de $138.6 \mathrm{mmol}$ de PETN/g.h.

\section{Discusión}

Identificación de las cepas degradadoras de PETN.- La secuencia parcial del gen $R N A r 16 S$ de la cepa J8A2 permitió ubicarla dentro del clado de la especie Bacillus licheniformis con 94\% de probabilidad según el árbol filogenético (Fig. 1). Bacillus licheniformis forma parte del grupo filogenético 1 determinado en base a la secuencia del gen $R N A r 16 S$ y que incluye a especies como B. subtilis, B. pumilus, B. amyloliquefaciens, entre otras (Wang ySun 2009). Por otro lado, según las características fenotípicas de la cepa J8A2 y las registradas en el Manual de Bacteriología Sistemática de Bergey (De Vos et al. 2009), el uso de citrato como fuente de carbono, el crecimiento en $7 \%$ de $\mathrm{NaCl}$ y el crecimiento en anaerobiosis lo relacionó presuntivamente con B. licheniformis, sin embargo, la coloración Gram negativa, la ausencia de actividad amilolítica y la inhibición del crecimiento a $50^{\circ} \mathrm{C}$ no permitió relacionarlo taxonómicamente con ninguna especie.

Asimismo, la secuencia del gen $R N A r 16 S$ de la cepa M1B sólo permitió identificarla dentro del género Enterobacter pero no especie, ya que este marcador no es suficiente para diferenciar especies de la familia Enterobacteriaceae (Spröer et al. 1999). La cepa M1B concordó fenotípicamente con las características generales del género Enterobacter del Manual de Bacteriología Sistemática de Bergey (Brenner et al. 2005). Sólo con Enterobacter cloacae y E. kobei compartió el mayor número de características: Voges-Proskauer negativo, indol negativo, arginina dehidrolasa y ornitina decarboxilasa. En conclusión, nuestros resultados indican que la cepa M1B es Enterobacter sp.

Cabe resaltar que el género Bacillus incluye especies Gram positivas, Gram negativas y Gram variables (De Vos et al. 2009). Entre las especies halofílicas Gram negativas se ha reportado a Bacillus horti (Yumoto 1998), así como se ha 
caracterizado cepas Gram negativas de este género con capacidad de degradar queratina, como Bacillus sp. PW-1 (Joshi 2007) que comparte las siguientes características fenotípicas con la cepa J8A2: motilidad, uso de citrato como única fuente carbono, sin actividad amilolítica, no produce $\mathrm{H}_{2} \mathrm{~S}$ y fermenta glucosa con producción de acidez y gas. Además, los cambios morfológicos de colonia registrados son características muy particulares dentro del grupo relacionado con Bacillus subtilis y sobre todo en el caso de Bacillus licheniformis (De Vos et al. 2009, Waldeck 2006, Carlisle 1989).

Finalmente, podemos afirmar que la cepa J8A2 mantiene una similitud significativa con la especie Bacillus licheniformis a nivel de la secuencia parcial del gen $R N A r 16 S$, sin embargo algunas de las características fenotípicas son incongruentes con dicha especie por lo que la cepa J8A2 ha sido designada como Bacillus sp.

Evaluación de la cinética de degradación de PETN.- Las velocidades específicas de crecimiento de las cepas M1B y J8A2, $0.057 \mathrm{~h}^{-1}$ y $0.042 \mathrm{~h}^{-1}$ respectivamente, son comparables entre sí; mientras que la velocidad específica de crecimiento de $E$. cloacae PB2 fuede $0.112 \mathrm{~h}^{-1}$ (Binks et al. 1996), 1.9 veces más que M1B y 2.7 veces más que J8A2. Sin embargo, el rendimiento de J8A2, $39.7 \mathrm{~g}$ de proteína por mol de PETN, fue 1.8 veces más que $\mathrm{M} 1 \mathrm{~B}$ que significó mayor cantidad de grupos $\mathrm{NO}_{2}$ del explosivo incorporado al metabolismo de J8A2.

Por otro lado, E. cloacae $\mathrm{PB} 2$ registró un rendimiento de 43.6 \pm 2.4 g de proteína por mol de PETN (French et al. 1998), 1.09 veces más que el rendimiento obtenido por J8A2, lo cual los hace comparables en rendimiento. Sin embargo, las condiciones de incubación ensayadas fueron de $30^{\circ} \mathrm{C}$ de temperatura y $170 \mathrm{rpm}$, mientras que en el presente estudio se realizó a 28 ${ }^{\circ} \mathrm{C}$ y $200 \mathrm{rpm}$.

Ambas cepas redujeron a la mitad la concentración de PETN en un promedio de 35 horas, mientras que E. cloacae PB2 es capaz de reducir la misma cantidad en menos de 30 horas; sin embargo, se debe tener en cuenta que la concentración inicial en el presente estudio fue de $158 \mathrm{mg} / \mathrm{L}$, mientras que en la cinética de E. cloacae PB2 fue de $32 \mathrm{mg} / \mathrm{L}$ aproximadamente.

Las velocidades específicas de degradación obtenidas, 1.16 mmol de PETN/g de proteína.h para M1B y $0.72 \mathrm{mmol}$ de $\mathrm{PETN} / \mathrm{g}$ de proteína.h muestran que la cepa M1B fue capaz de convertir en proteína 1.6 veces más PETN por hora que la cepa J8A2, mientras que Enterobacter cloacae PB2 mostró un velocidad específica de degradación de $1.03 \mathrm{mmol}$ de PETN/g de proteína.h similar al de M1B, pero 1.4 veces más que J8A2.

La enzima PETN reductasa posee un grupo prostético de FMN (Flavin mononucleótido), común en la familia de las flavoenzimas y evidencia un color amarillo claro con la mayor absorbancia a $470 \mathrm{~nm}$ cuando es purificado. Esta característica se ha reportado en el estudio de las old yellow enzime (OYE) homólogos de Pseudomonas putida, XenA, XenB, XenC, XenE y XenF, relacionados con la degradación de compuestos nitroaromáticos como TNT (Van Dillewijnet al. 2008). Además, el ciclo catalítico de la PETN reductasa comprende una reacción de dos pasos. La primera es reductiva, donde la enzima es reducida por el NADPH hasta obtener la forma dihidroquinona del grupo prostético FMN de la enzima. Luego, en la segunda reacción oxidativa la FMN es oxidada por grupos $\mathrm{NO}_{2}$ del explosivo (Khan et al. 2002, Khan et al. 2004). Por otro lado, otro reporte afirma que los cristales de PETN en estado reducido son incoloros y en su estado oxidado son amarillos brillantes (Khan et al. 2005), por lo que el PETN reducido no aporta cambio de color en el medio. Entonces, el cambio de color del cultivo podría deberse a la producción de flavoenzimas y la completa oxidación de los grupos prostéticos FMN que en consecuencia pigmentan el medio de amarillo.

Evaluación de la actividad PETN reductasa.- Tanto la velocidad específica de liberación de $\mathrm{NO}^{-2}$ como la velocidad específica de oxidación de NADPH, $4.6 \mu \mathrm{mol} / \mathrm{min}$.mg de proteína y $4.7 \mu \mathrm{mol} / \mathrm{min}$.mg de proteína demuestran que se requiere de una molécula de NADPH para liberar una molécula de nitrito, tal y como se ha reportado con E. cloacae PB2 que mostró 4.6 $\mu \mathrm{mol} / \mathrm{min}$.mg de velocidad específica de liberación de $\mathrm{NO}^{-2}$ y 4.2 $\mu \mathrm{mol} / \mathrm{min}$.mg de velocidad específica de oxidación de NADPH. Esto demuestra la dependencia de un cofactor de esta naturaleza para la degradación de PETN, lo cual fue confirmado por los controles sin NADPH que registraron velocidad específica de liberación de $\mathrm{NO}_{2}$ de $1.5 \mu \mathrm{mol} / \mathrm{min} . \mathrm{mg}, 3.1$ veces menos que los ensayos con NADPH.

La actividad específica por proteína (U/g) de E. cloacae PB2 sugiere una liberación lenta de nitritos al medio durante su crecimiento, pero su velocidad específica de crecimiento y degradación sugieren una incorporación rápida de estos a su metabolismo, lo cual podría impedir una acumulación de nitritos en el medio disminuyendo así la concentración y toxicidad de éste (Tilak et al. 2007, Cabrera \& Velázquez 1998). En cambio, la cepa J8A2 mostró un comportamiento contrario reflejado en una mayor actividad específica por proteína $(\mathrm{U} / \mathrm{g})$, pero una menor velocidad específica de crecimiento y degradación por la acumulación de nitritos en el medio. Esto explicaría la limitación de crecimiento de la cepas J8A2 y M1B durante las cinéticas ensayadas (Figs. 2 y 3) alcanzando a producir sólo 16.74g/L de proteína total.

\section{Agradecimientos}

El presente trabajo fue financiado parcialmente por la Universidad Nacional Mayor de San Marcos a través del Vicerrectorado de Investigación con el proyecto № 131001161.

\section{Literatura citada}

Altschul S., W. Gish, W. Miller, E. Myers \& D. Lipman.1990. Basic local alignment search tool. Journal of Molecular Biology.215(3): 403-410.

Binks P., C. French, S. Nicklin \& N. Bruce. 1996. Degradation of Pentaerythritol Tetranitrate by Enterobacter cloacae PB2. Applied and Environmental Microbiology. 62(4): 1214-1219.

Bradford M. 1976. A rapid and sensitive method for quantitation of microgram quantities of protein utilizing the principle of protein-dyebinding.Analytical Biochemical. 72:248-54. DOI 10.1016/00032697(76)90527-3

Brenner D., N. Krieg \& J.Staley. 2005. Bergey's manual of systematic bacteriology (Volume two: The proteobacteria part B the Gammaproteobacteria). Editor in Chief: George M. Garrity. Segundaedición. London New York: Springer Dordrecht Heidelberg, 1136 p. ISBN 0-387-95040-0.

Cabrera J.M. \& R.O. Velázquez. 1998. Intoxicación por sustancias metahemoglobinizantes. Estudio retrospectivo de 39 pacientes. Revista Cubana Medicina. 37(2):77-82.

Carlisle G.E. \& J.O. Falkinham III. 1989. Enzyme activities and antibiotic susceptibility of colonial variants of Bacillus subtilis and Bacillus licheniformis. Applied and Environmental Microbiology. 55(11): 3026-3028. 
De Angelis K.M., P. D’Haeseleer, D. Chivian, J. L. Fortney, J. Khudyakov, B. Simmons, H. Woo, A. P. Arkin, K. Walston Davenport, L. Goodwin, A. Chen, N. Ivanova, N.C.Kyrpides, K. Mavromatis, T. Woyke \& T.C. Hazen. 2011. Complete genome sequence of "Enterobacter lignolyticus" SCF1. Standards in Genomic Sciences.5:69-85. doi: $10.4056 /$ sigs. 2104875

De Vos P.,G. Garrity, D. Jones, N. Krieg, W. Ludwig, F. Rainey, K. H. Schleifer $\&$ W. Whitman. 2009. Bergey's manual of systematic bacteriology (Volume three: The firmicutes). Editor in Chief: Michael Goodfellow.Segundaedición. London New York: Springer Dordrecht Heidelberg, 1450 p. ISBN: 978-0-387-95041-9.

Esteve-Nuñez A., A. Caballero \& J. Ramos. 2001. Biological degradation of 2,4,6 trinitrotoluene. Microbiology and Molecular Biology Reviews.65(3):335-352. DOI 10.1128/MMBR.65.3.335-352.2001

Estudios Mineros del Perú S.A.C. 2005. (En línea). Manual de Minería.<http:// ingenierosdeminas.org/biblioteca_digital/libros/Manual_Mineria. pdf>. Acceso: 15/01/2011.

French C., S. Nicklin \& N. Bruce. 1998. Aerobic degradation of 2,4,6-trinitrotoluene by Enterobacter cloacae PB2 and by pentaerythritol tetranitrate reductase. Applied and Environmental Microbiology. 64(8): 2864-2868.

French C., S. Nicklin \& N. Bruce. 1996. Sequence and properties of pentaerythritol tetranitrate reductase from Enterobacter cloacae PB2. Journal of Bacteriology. 178(22): 6623-6627.

Foltz M.F. 2012. (En línea). Aging of Pentaerythritol Tetranitrate (PETN).U.S. Department of Energy and Lawrence Livermore National Laboratory.<https://e-reports-ext.llnl.gov/pdf/372573.pdf>. Acceso 13/05/2012.

Global Security. 2011. (En línea). Military explosives: PETN [Pentaerythritol tetranitrate] <http://www.globalsecurity.org/military/systems/munitions/explosives-nitrate-petn.htm>Acceso 20/02/2011.

Hall T. 1999. BioEdit: a user-friendly biological sequence alignment editor and analysis program for Windows 95/98/NT. Nuclei.Acids. Symp. 41: 95-98.

Ibeanusi V., J. Yassin, S. Houston, D. Doss \& B. Coley. 2009. Sequential anaerobic-aerobic degradation of munitions waste. Biotechnology Letters. 31: 65-69. DOI 10.1007/s10529-008-9828-9

Jaw K.S. \& J.S. Lee. 2008. Thermal behaviors of PETN base polymer bonded explosives. Journal of Thermal Analysis and Calorimetry. 93(3): 953-957. DOI 10.1007/s10973-006-7736-6

Joshi S.G., M.M. Tejashwini, N. Revati, R. Sridevi \& D. Roma. 2007. Isolation, identification and characterization of a feather degrading bacterium. International Journal of Poultry Science. 6(9): 689-693.

Khan H., R. Harris, T. Barna, D. Craig, N. Bruce, A. Munro, P.Moody $\&$ N. Scrutton. 2002. Kinetic and structural basis of reactivity of Pentaerythritol Tetranitrate reductase with NADPH, 2-Cyclohexenone,Nitroesters and Nitroaromatic explosives. The Journal of Biological Chemistry. 277(24): 21906-21912. doi: 10.1074/jbc.M200637200

Khan H., T. Barna, R. Harris, N. Bruce, I. Barsukov, A. Munro, P. Moody \& N. Scrutton. 2004. Atomic resolution structures and solution behavior of enzyme-substrate complexes of Enterobacter cloacae PB2 Pentaerythritol Tetranitrate reductase. The Journal of Biological Chemistry. 279 (29): 30563-30572. doi: 10.1074/jbc.M403541200

Khan H., T. Barna, N. Bruce, M. Andrew, D. Leys \& N. Scrutton. 2005. Proton transfer in the oxidative half-reaction of Pentaerythritol Tetranitrate reductase. FEBS Journal.272: 4660-4671. DOI: 10.1111/j.1742$4658.2005 .04875 . x$
Singh B., J. Kaur \& K. Singh. 2012. Microbial remediation of explosive waste. Critical Reviews in Microbiology. 38(2): 152-167. doi:10.3109/1 040841X.2011.640979

Kumar S., N. Masatoshi, Du. Joel \& T. Koichiro. 2008. MEGA: A biologistcentric software for evolutionary analysis of DNA and protein sequences. Briefings in Bioinformatics.9(4): 299-306. doi: 10.1093/ bib/bbn017

Nyanhongo G., A. Nina, M. Ortner, W. Steiner \& G. Guebitz. 2008. A novel environmentally friendly 2,4,6-trinitrotoluene (TNT) based explosive. Macedonian Journal of Chemistry and Chemical Engineering. 27(2): 107-116.

Park C., T.H. Kim, S. Kim,S.W. Kim, J. Lee \& S.H. Kim. 2003. Optimization for biodegradation of 2,4,6-trinitrotoluene (TNT) by Pseudomonas putida. Journal of Bioscience and Bioengineering.95(6): 567-571. DOI 10.1016/S1389-1723(03)80163-9

SINIA (Sistema Nacional de Información Ambiental). 2007. (En línea). Plan nacional de implementación del Convenio de Estocolmo sobre los contaminantes orgánicos persistentes <http://sinia.minam.gob.pe/ index.php?idElementoInformacion=2>.Acceso: 15/03/11.

Spröer C., U. Mendrock, J. Swiderski, E. Lang \& E. Stackebrandt. 1999. The phylogenetic position of Serratia, Buttiauxella and some other genera of the family Enterobacteriaceae. International Journal of Systematic Bacteriology 49: 1433-1438.

Tamura K., D. Peterson, N. Peterson, G. Stecher, M. Nei, S. Kumar. 2011. MEGA5: Molecular Evolutionary Genetics Analysis Using Maximum Likelihood, Evolutionary Distance, and Maximum Parsimony Methods. Molecular Biology and Evolution 28(10):2731-2739. doi: $10.1093 / \mathrm{molbev} / \mathrm{msr} 121$

Tilak K.S., K. Veeraiah \& J.M.P. Raju. 2007. Effects of ammonia, nitrite and nitrate on hemoglobin content and oxygen consumption of freshwater fish, Cyprinuscarpio (Linnaeus). Journal of Environmental Biology 28(1): 45-47.

Trott S., S. Nishino, J. Hawari \& J. Spain. 2003. Biodegradation of the nitramine explosive CL-20. Applied and Environmental Microbiology 69(3): 1871-1874. doi: 10.1128/AEM.69.3.1871-1874.2003

Van Dillewijn P., R.M. Wittich, A. Caballero \& J.L. Ramos. 2008. Subfunctionality of hydride transferases of the old yellow enzyme family of flavoproteins of Pseudomonas putida. Applied and environmental microbiology 74(21): 6703-6708. doi: 10.1128/AEM.00386-08

Waldeck J., G. Daum, B. Bisping \& F. Meinhardt. 2006. Isolation and molecular characterization of chitinase-deficient Bacillus licheniformis strains capable of deproteinization of shrimp shell waste to obtain highly viscous chitin. Applied and Environmental Microbiology 72(12): 7879-7885. doi: 10.1128/AEM.00938-06

Wang W. \& M. Sun. 2009. Phylogenetic relationships between bacillus species and related genera inferred from 16S rDNA sequences. Brazilian Journal of Microbiology40: 505-521. DOI 10.1590/S151783822009000300013

Weisburg W.G., S.M. Barns, D.A. Pelletier \& D.J. Lane. 1991. 16S Ribosomal DNA amplification for phylogenetic study. Journal of Bacteriology 173(2:697-703.

White G., J. Snape \& S. Nicklin. 1996. Biodegradation of Glycerol Trinitrate and Pentaerythritol Tetranitrate by Agrobacterium radiobacter. Applied and Environmental Microbiology 62(2): 637-642.

Yumoto I., K. Yamazaki, T. Sawabe, K. Nakano, K. Kawasaki, Y. Ezura \& H. Shinano. 1998. Bacillus horti sp. nov., a new Gram-negative a alkalophilic bacillus. International Journal of Systematic Bacteriology 48: 565-571. 